\title{
AMENDMENTS
}

\section{Publisher Correction: Shared proteomic effects of cerebral atherosclerosis and Alzheimer's disease on the human brain}

\author{
Aliza P. Wingo (D), Wen Fan, Duc M. Duong, Ekaterina S. Gerasimov, Eric B. Dammer D, Yue Liu, Nadia V. Harerimana, \\ Bartholomew White, Madhav Thambisetty, Juan C. Troncoso, Namhee Kim, Julie A. Schneider, Ihab M. Hajjar, \\ James J. Lah, David A. Bennett, Nicholas T. Seyfried (D), Allan I. Levey (D) and Thomas S. Wingo (D)
}

Correction to: Nature Neuroscience https://doi.org/10.1038/s41593-020-0635-5, published online 18 May 2020.

Before print:

In the version of this article initially published online, a word was erroneously added to the second sentence of the abstract. The sentence should read, "We performed proteomic sequencing of dorsolateral prefrontal cortex in 438 older individuals and found associations between cerebral atherosclerosis and reduced synaptic signaling and RNA splicing and increased oligodendrocyte development and myelination." The error has been corrected in the print, PDF and HTML versions of this article.

Published online: 23 June 2020

https://doi.org/10.1038/s41593-020-0662-2

( ) The Author(s), under exclusive licence to Springer Nature America, Inc. 2020

\section{Publisher Correction: Dynamic regulation of Z-DNA in the mouse prefrontal cortex by the RNA-editing enzyme Adar1 is required for fear extinction}

Paul R. Marshall, Qiongyi Zhao D, Xiang Li, Wei Wei, Ambika Periyakaruppiah, Esmi L. Zajaczkowski, Laura J. Leighton, Sachithrani U. Madugalle, Dean Basic, Ziqi Wang, Jiayu Yin, Wei-Siang Liau, Ankita Gupte, Carl R. Walkley and Timothy W. Bredy (D)

Correction to: Nature Neuroscience https://doi.org/10.1038/s41593-020-0627-5, published online 4 May 2020.

In the version of this article initially published, the abbreviation "s.e.m" (standard error of the mean) was incorrectly added to $x$-axis legends in Figs. 2b,f; $4 b-g ; 6 a, e, i$; and $7 b$,e. Additionally, the fourth sentence of the first paragraph should have read, "Given this, there is...." The errors have been corrected in the print, HTML and PDF versions of the article.

Published online: 26 June 2020

https://doi.org/10.1038/s41593-020-0669-8

(c) The Author(s), under exclusive licence to Springer Nature America, Inc. 2020 